\title{
An Unusual Case of Facial Steatocystoma Multiplex: A Clinicopathologic and Dermoscopic Report
}

\author{
Aseem Sharma Sandip Agrawal Rachita Dhurat Dipti Shukla \\ Tejas Vishwanath \\ Department of Dermatology, Lokmanya Tilak Municipal Medical College (LTMMC) \\ and Sion General Hospital, Mumbai, India
}

Keywords

Facial steatocystoma multiplex $\cdot$ Sebaceous gland $\cdot$ Hamartoma $\cdot$ Dermoscopy

\section{Abstract}

Background: Steatocystoma multiplex is a benign skin disorder originating from the sebaceous and nevoid ducts. Commonly classified under hamartomas, they are distributed over the trunk, neck, axillae, and groin. Methods: A 28-year-old male patient complained of multiple, asymptomatic skin-colored nodules over the face of 10 -year duration. Clinical examination confirmed the historic findings of nontender, polysized, flesh-colored papules and nodules over the said distribution. Results: On histopathology, a cyst was noted in the mid-dermis, lined by stratified squamous, agranular epithelium, which contained degenerated keratin. Nonpolarized dermoscopy showed a structureless, cream-colored area, and polarized dermoscopy revealed a distinctive, well-circumscribed, yellowish hue which was superimposed over the facial pseudoreticular pigmentary pattern. The findings were compatible with steatocystoma multiplex, and the patient was taken up for radiofrequency ablation. Conclusion: Herein, we report a rare variant of steatocystoma multiplex limited to the face and scalp subjected to dermatoscopy and characteristic histological correlation. To the best of our knowledge and following a literature search, dermoscopic features of this condition have not been reported thus far. 


\section{Introduction}

Steatocystoma multiplex (SCM) is a rare, hamartomatous disorder of the pilosebaceous unit characterized by sebum-containing cysts in the dermis. First described by Jamieson in 1873 and coined by Pringle in 1899, the nosology literally translates to "a bag of fat." SCM is synonymous with polycystic disease of the epidermis and sebocystomatosis [1]. They can occur as isolated, singular lesions and, in that scenario, the term steatocystoma simplex is used. The existing classification of SCM includes generalized, localized, acral, facial, flexural, suppurative, and syndromal types [1,2]. This case presented with lesions confined to the head and neck region, with classic histological findings. We propose new dermoscopic findings in SCM which are, hitherto, unreported.

\section{Case Report}

A 28-year-old male patient presented with multiple, asymptomatic skin-colored nodules involving the forehead and scalp of 10-year duration. The lesions first appeared on the face and then gradually progressed to involve the scalp. He gave history of freshly occurring lesions and progression of existing lesions, both in terms of size and number. On examination, there were multiple, nonpruritic, nontender, flesh-colored papules to nodules ranging from 0.3 to $1 \mathrm{~cm}$ in diameter (Fig. 1,2). They exhibited a smooth surface and soft consistency, and revealed yellowish, oily-to-cheesy material (depending on the duration of the lesion) expressed through the punctum, wherever present. There was no history of similar lesions in his family. A punch incisional biopsy through the lesion was taken, sectioned, and stained with hematoxylin and eosin (H\&E). Histopathology revealed a cyst in the mid-dermis, characterized by 2 - to 3-layered stratified squamous epithelium with absence of a granular layer, an eosinophilic, crenulated cuticle showing sharp invaginations into the lumen, and amorphous keratinous, eosinophilic material in the cyst cavity (Fig. 3, 4). These clinical and histological findings were compatible with SCM. Informed consent was sought and recorded from
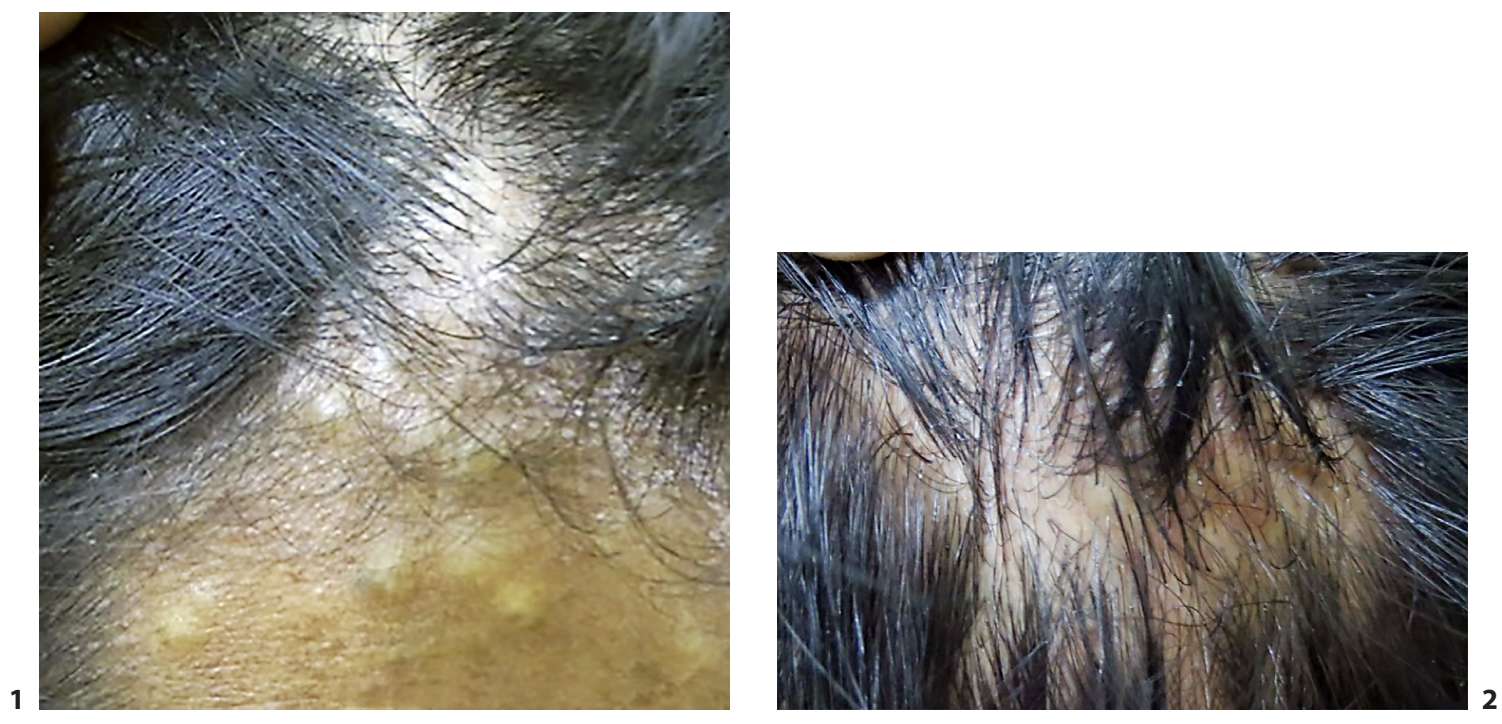

Fig. 1. Multiple creamy, yellowish-colored papules and nodules ranging from 0.3 to $1 \mathrm{~cm}$ in size over the forehead.

Fig. 2. Flesh-colored to yellow papules and nodules over the scalp. 
Sharma et al.: Clinicopathologic and Dermoscopic Report of Facial Steatocystoma Multiplex
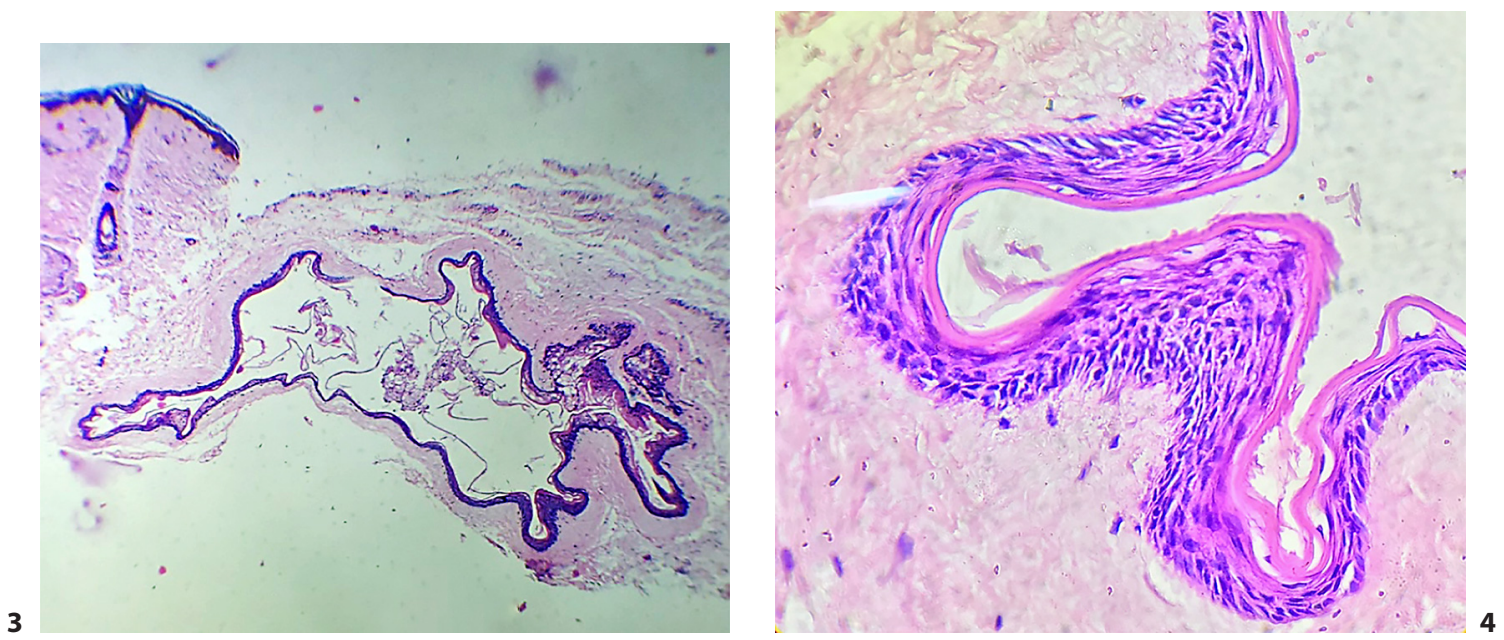

Fig. 3. An epithelial cyst lined with a thin wall in the mid-dermis. A sebaceous gland is attached to the cyst wall. The cyst cavity shows keratinous debris. H\&E. $\times 10$.

Fig. 4. A folded cyst wall consisting of several layers of stratified epithelium with absence of the granular layer. H\&E. $\times 40$.
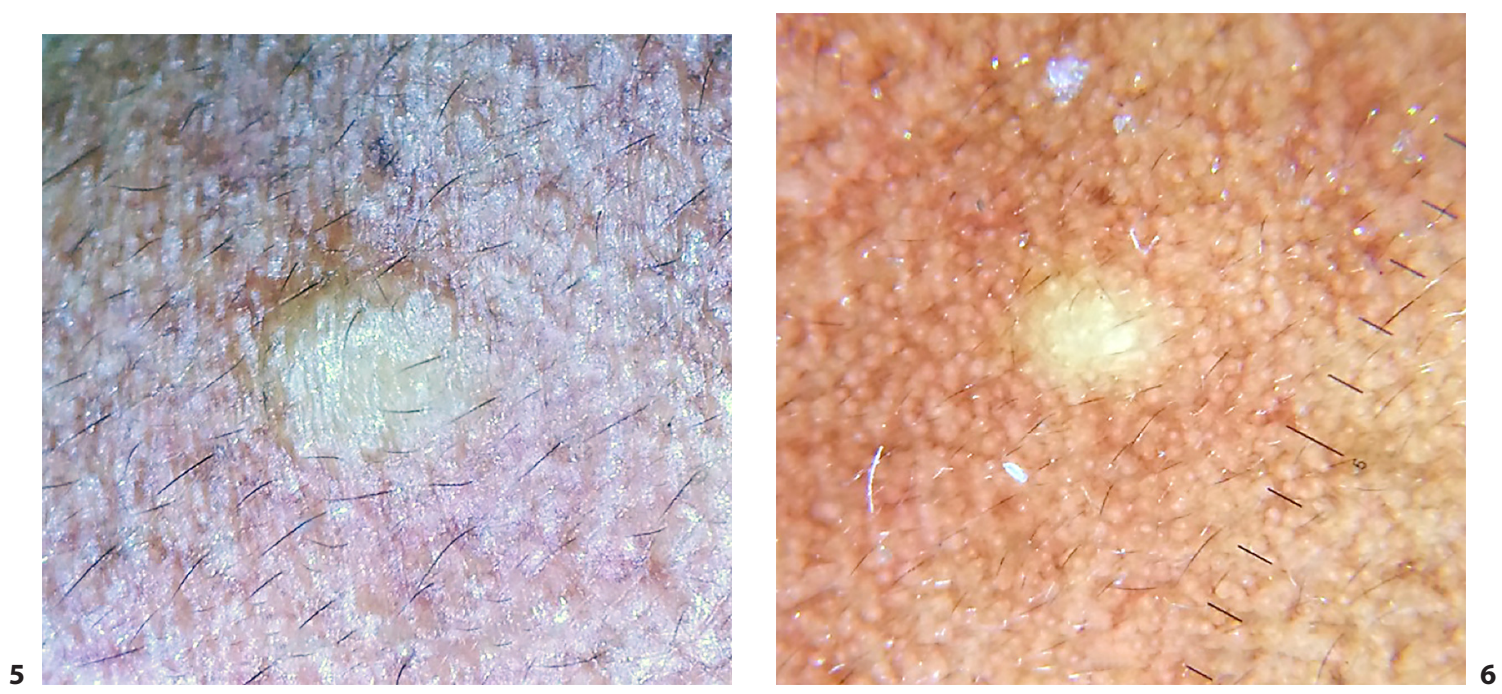

Fig. 5. Nonpolarized dermoscopy showing a circumscribed yellowish structureless area with diffuse margins, surrounded by vellus hairs.

Fig. 6. Polarized dermoscopy shows a central yellow glow with preserved pseudoreticular pigmentary pattern over the facial papule.

the subject. The study protocol was approved by the Ethics Committee, Staff Research Society, Lokmanya Tilak Municipal Medical College and General Hospital, Mumbai.

Dermatoscopy was performed, both polarized and nonpolarized. The latter showed a yellowish structureless area with diffuse margins. However, on polarized dermoscopy, lesions showed a distinctive yellowish glow, surrounded by a darker rim, over a backdrop of a pseudoreticular pigmentary pattern (Fig. 5, 6). The yellow structureless area correlated with sebum contained in the cyst cavity. Vellus hairs, wherever entrapped in the cystic cavity, imparted a greenish tinge to the yellow area. The patient was posted for radiofrequency ablation. 
Sharma et al.: Clinicopathologic and Dermoscopic Report of Facial Steatocystoma Multiplex

\section{Discussion}

SCM is an uncommon disorder of the pilosebaceous unit characterized by sebumcontaining dermal cysts. It is inherited in an autosomal dominant fashion, albeit most cases occur sporadically [2,3]. Clinically, it manifests as skin-colored to yellowish, elastic or firm, cystic or dome-shaped papules or nodules showing varied distribution. Areas of involvement include the trunk, neck, the groin, scalp, and proximal extremities. However, uncommon sites may be affected, viz., face, glutei, breasts, or flexures. The lesions are usually devoid of a central punctum, and yellowish, cheesy, semisolid secretion can be expressed through the same.

The differential diagnoses include epidermal and epidermoid cysts, eruptive vellus hair cysts, neurofibromatosis, lipomatosis, milia, sebaceous hyperplasia, sebaceous adenomas, and xanthomatosis. The term "sebaceous cyst" is often used interchangeably with epidermal cyst, which is essentially a misnomer. In fact, SCM is the only cyst with a true sebaceous origin. On histopathology, SCM shows flattened sebaceous lobules located close to the cystic wall. Rarely, a sebaceous adenoma may form a part of the wall and is named steatosebocystadenoma. The cyst wall is composed of stratified, squamous epithelium, with the granular layer characteristically absent. The cystic lumen is lined by a thick, homogeneous, eosinophilic cuticle. The intricate folding pattern and invagination of the cuticle into the lumen is attributed to the collapse of the cyst wall during processing and removal of sebum and lipids. Single biopsies may not show pathognomonic features, and, hence, serial biopsies should be performed in case of suspicion. Untreated SCM can cause sequelae of scarring, both hypertrophic and atrophic, akin to nodulocystic acne and hidradenitis suppurativa [1, 4].

Electron microscopy has proven to be a useful tool in understanding the histogenesis of SCM. The differentiation of the cyst wall is in the direction of the sebaceous duct. A thin, horny layer lining the lumen comprises elongated, flattened cells followed by a layer of stratified epithelium with columnar cells at the base (in early lesions), which are replaced by shorter cells in chronic lesions. The undulation of the horny cuticle and immature sebocytes in the wall help differentiate it from other conditions. Early lesions also show a few keratohyaline granules, which regress in chronic lesions, translating to the characteristic absence of the granular layer [5]. This sebaceous differentiation may often coexist with trichilemmal, pilomatricomal, and infundibular keratinization, causing SCM to coexist with eruptive vellus hair cysts (EVHC), trichofolliculomas, trichostasis spinulosa, pilomatrixomas, and epidermoid cysts, respectively. These can occur concomitantly, asynchronously, or even manifest as hybrid cysts [6].

SCM and EVHC have traditionally been thought of as variants of the same disease, one single entity, and close associations by various schools of thought. The similarities include inheritance pattern, age at onset, site of distribution, morphology, and histological localization to the mid-dermis. They differ in terms of cyst contents - oily, cheesy material in SCM versus a vellus hair strand mesh in EVHC, with characteristically different histologies. The cyst wall is associated with sebaceous lobules or mature glands in SCM, and vellus hair follicles in EVHC $[6,7]$. This author deduces that EVHC and SCM are cystic offshoots from a common origin - the pilosebaceous unit, subjected to distinct patterns of differentiation, making them exclusive, related entities.

Additionally, immunohistochemistry done on squamous epithelium of these cysts has further helped delineate these closely related entities on the basis of varying keratin expression patterns. SCM show expression of K7 and K10, EVHC show expression of K17, and epidermoid cysts show K10 [6-8].

Syndromal SCM comprises a few syndromes associated with SCM, which may be of premonitory significance. Expression of K17 has been implicated in pachyonychia congenita, 
characterized by oral leukokeratosis, palmoplantar keratoderma, follicular hyperkeratosis, generalized SCM, and EVHC [9]. Along with EVHC, they are also associated with the X-linked recessive Lowe syndrome or oculocerebrorenal syndrome, with cataracts, hypotonia, and renal dysfunction [10]. The Gardner syndrome, known as familial adenomatous polyposis, presents with a plethora of cysts, including epidermal, dermoid, infundibular, and steatocystomas [11]. Steatocystomas may also be present as a part of the Noonan syndrome or LEOPARD syndrome with lentiginosis, ocular hypertelorism, stunted growth, genital anomalies, and deafness [12].SCM has also been mentioned in conjunction with the Alagille-Watson syndrome affecting the heart, musculoskeletal and hepatorenal systems [12], and the Favre-Racouchot syndrome, featuring solar elastosis, comedones, and infundibulofollicular cysts [13].

Treatment is primarily interventional, comprising aspiration, cold steel surgery, cryotherapy, radiofrequency ablation, and ablative lasers. Few authors attempted oral retinoids, with limited success and a high recurrence rate $[14,15]$. Aspiration is a simple procedure, best suited for small lesions. Recurrence rates are high as the cyst wall is left intact. Cryotherapy is suited for larger lesions, even calcified ones, but it is associated with procedural pain, blister formation, hypopigmentation, and recurrence. Ablative lasers like carbon dioxide successfully vaporize the cyst wall and remnants but may cause scarring and hypopigmentation. Additionally, the therapy is not cost effective. Radiofrequency ablation, combined with surgical involution and excision of the cyst wall, has been reviewed as the method of choice. The risk of recurrence is almost negligible with this particular modality [16].

The occurrence of the facial variant of SCM has been described and reported in the global literature on a number of occasions. The same holds true for its coexistence with EVHC, trichofolliculomas, and epidermoid cysts [17-24]. However, this study additionally delineates the dermatoscopic correlation with histopathology.

\section{Conclusion}

SCM is a true sebaceous cyst, causing significant cosmetic distress, and is poorly responsive to conventional therapeutic modalities. The facial, papular variant has been described previously in the literature, prior to this, albeit this report describes associated dermoscopic features.

\section{Statement of Ethics}

Informed consent was sought and recorded from the subject. The study protocol was approved by the Ethics Committee, Staff Research Society, and Lokmanya Tilak Municipal Medical College and General Hospital, Mumbai.

\section{Disclosure Statement}

The authors declare no conflicts of interest. 
Sharma et al.: Clinicopathologic and Dermoscopic Report of Facial Steatocystoma Multiplex

\section{References}

1 Alsabbagh MM: Steatocystoma multiplex: A review. J Dermatol Dermatol Surg 2016;20:91-99.

-2 Kamra HT, Gadgil PA, Ovhal AG, Narkhede RR: Steatocystoma multiplex - a rare genetic disorder: a case report and review of the literature. J Clin Diagn Res 2013;7:166-168.

3 Cho S, Chang SE, Choi JH, et al: Clinical and histologic features of 64 cases of steatocystoma multiplex. J Dermatol 2002;29:152-156.

4 Thomas VD, Swanson NA, Lee KK: Benign epithelial tumors, hamartomas, and hyperplasias; in Wolff K, Goldsmith LA, Katz SI, Gilchrest BA, Paller AS, Leffell DJ (eds): Fitzpatrick's Dermatology in General Medicine, ed 7. New York, McGraw-Hill, 2008, pp 1065-1066.

5 Plewig G, Wolff HH, Braun-Falco 0: Steatocystoma multiplex: anatomic reevaluation, electron microscopy, and autoradiography. Arch Dermatol Res 1982;272:363.

6 Tomkova H, Fujimoto W, Arata J: Expression of keratins (K10 and K17) in steatocystoma multiplex, eruptive vellus hair cysts, and epidermoid and trichilemmal cysts. Am J Dermatopathol 1997;19:250.

-7 Papakonstantinou E, Franke I, Gollnick H: Facial steatocystoma multiplex combined with eruptive vellus hair cysts: a hybrid? J Eur Acad Dermatol Venereol 2015;29:2051-2053.

-8 Kurokawa I, Takahashi K, Moll I, Moll R: Expression of keratins in cutaneous epithelial tumors and related disorders - distribution and clinical significance. Exp Dermatol 2011;20:217-228.

-9 Hodes ME, Norin AL: Pachyonychia congenita and steatocystoma multiplex. Clin Genet 1977;11:359-364.

10 Bhushan P, Singh A: Facial variant of eruptive vellus hair cyst. Indian J Dermatol Venereol Leprol 2014;80: 96-98.

-11 Leppard B, Thompson HR: Gardner's syndrome and steatocystoma multiplex. Two unusual genetically determined conditions occurring in same patient. J Med Genet 1976;13:407-411.

12 Mahmood M, Sabbagh A: Steatocystoma multiplex: a review. J Dermatol Dermatol Surg 2016;20:91-99.

13 Kulkarni V: Favre-Racouchot syndrome. Indian J Dermatol Venereol Leprol 1991;57:244-245.

14 Rosen BL, Brodkin RH: Isotretinoin in the treatment of steatocystoma multiplex: a possible adverse reaction. Cutis 1986;37:115-120.

15 Apaydin R, Bilen N, Bayramgürler D, Basdas F, Harova G, Dökmeci S: Steatocystoma multiplex suppurativum: oral isotretinoin treatment combined with cryotherapy. Australas J Dermatol 2000;41:98-100.

16 Choudhary S, Koley S, Salodkar A: A modified surgical technique for steatocystoma multiplex. J Cutan Aesthet Surg 2010;3:25-28.

17 Hansen KK, Troy JL, Fairley JA: Multiple papules of the scalp and forehead: steatocystoma multiplex (facial papular variant). Arch Dermatol 1995;131:835-838.

18 Albayrak H, Anık ME, Güvenç SC, Yıldırım U, Aliağaoğlu C: A rare form of steatocystoma multiplex predominantly located on the face. Düzce Tıp Fakültesi Dergisi 2008;3:57-59.

19 Düzova N, Șentürk GB: Suggestion for the treatment of steatocystoma multiplex located exclusively on the face. Int J Dermatol 2004;43:60-62.

20 Derya U, Bilal S, Zeynep MA, Veysi F, Uğur F, Mustafa A: A rare case of facial steatocystoma multiplex. Acta Dermatovenerol Croat 2013;21:205-206.

21 Requena L, Martin L, Renedo G, Arias D, Espinel ML, de Castro A: A facial variant of steatocystoma multiplex. Cutis 1993;51:449-452.

-22 Sardana K, Sharma RC, Mahajan S: Facial steatocystoma multiplex associated with pilar cyst and bilateral preauricular sinus. J Dermatol 2002;29:157-159.

23 Aloi F, Tomasini C: Eruptive vellus hair cysts: a facial variant. G Ital Dermatol Venereol 1990;125:515-518.

24 Lew BL, Lee MH, Haw CR: Unilateral eruptive vellus hair cysts occurring on the face. J Eur Acad Dermatol Venereol 2006;20:1314-1316. 